\title{
Clinico-mycological study of dermatophytosis and their antifungal susceptibility, a hospital based study
}

\author{
Pradhan MB' ${ }^{1}$, Paudel V1 \\ ${ }^{1}$ Department of Dermatology, National Medical College Teaching Hospital, Birgunj, Nepal
}

\begin{abstract}
Introduction: Dermatophytosis refers to superficial fungal infections of keratinized tissues caused by keratinophilic dermatophytes. It is the most common of the superficial fungal infections. Nowadays, these fungal infections are at a rise and run a prolonged course despite of treatment due to resistance to conventional antifungal agents. There is a felt need to conduct an epidemiological studies to know the change in the pattern and causes of widespread resistance.

Objectives: This study was aimed at identifying clinico-mycological patterns of dermatophytic infections in patients attending the dermatology outpatient department of a tertiary care hospital in Birgunj and the adjoining areas.

Materials and Methods: The study included cases of clinically diagnosed dermatophytosis from the outpatient department of Dermatology of National Medical College, Birgunj. Clinical and epidemiological data were collected as per proforma and skin scraping, hair plucking, and nail clipping were done and materials were examined microscopically by $\mathrm{KOH}$ mount then cultured on Sabouraud dextrose agar and antifungal susceptibility were done by disk diffusion test.

Results: There were 349 patients recruited in the study, with a male: female ratio of 1.6:1. The most commonly affected age group was $20-29$ years (27.3\%). Tinea corporis was the most common type observed (38.1\%). Potassium hydroxide positivity was seen in 228 samples (65.3\%) and culture positivity was found in 202 samples (57.9\%). The most common species identified was Trichophyton rubrum (55\%). The most sensitive drug was itraconazole, and more number of resistances was noted with fluconazole.

Conclusion: Dermatophytic infection is ecumenical in distribution with increased frequency in tropical and subtropical countries with variable epidemiology. Inadequate and irregular use of antifungal drugs has led to the emergence of resistant strains, which cause poor treatment outcomes. Thus, it is essential to test for antifungal sensitivity to check for resistance to antifungals.
\end{abstract}

Key words: Antifungal Agents; Dermatomycoses; Tinea; Trichophyton rubrum

\section{Introduction}

$\mathrm{D}$ ermatophytes are fungi that cause superficial infections of the skin, hair, and nails that require keratin for growth. Dermatophytosis is commonly referred to as ringworm. Dermatophytes spread by direct contact from other people (anthropophilic), animals (zoophilic), and soil (geophilic), as well as indirectly from fomites. Microsporum, Trichophyton, and Epidermophyton species are the most common pathogens causing dermatophytic infection of hair, nail

Funding: No

Conflict of Interest: No

Address of Correspondence

Dr. Manish Bhakta Pradhan

Department of Dermatology and Venereology

National Medical College, Birgunj, Nepal

Phone: 9841007091

E-mail: pradhanmeister@gmail.com and skin. ${ }^{1}$ Clinically, tinea can be classified according to the site of involvement including tinea capitis, tinea corporis, tinea cruris, tinea pedis, tinea barbae, tinea manuum, tinea faciei and tinea unguium. ${ }^{2}$ The prevalence of superficial dermatophytic infections affects more than $20-25 \%$ of the world population and is one of the most frequent forms of infections. ${ }^{3}$

Submitted: $5^{\text {th }}$ December 2020

Accepted: $30^{\text {th }}$ January 2021

Published: $20^{\text {th }}$ February 2021

How to cite this article

Pradhan MB, Paudel V. Clinico-mycological study of dermatophytosis and their antifungal susceptibility, a hospital based study. Nepal Journal of Dermatology, Venereology \& Leprology 2021;19(1):30-6. https://doi.org/10.3126/njdvl. v19i1.34693.

Licensed under CC BY 4.0 International License which permits use, distribution and reproduction in any medium, provided the original work is properly cited. 
The frequencies of dermatophytes depends upon the community as well, mostly common with low socio economic communities. The expected lifetime risk of getting a dermatophyte infection is between 10 and $20 \%{ }^{4}$

There are several antifungal drugs used to treat dermatophytosis. Current therapeutics options for the treatment of superficial dermatophytosis rely on topical or oral antifungal drugs. Some infections respond well to topical antifungal therapy only, whereas others like tinea capitis, tinea unguium, and more extensive or severe types may require systemic therapy. ${ }^{5}$

The present study was undertaken to identify the clinic mycological pattern and to assess the antifungal susceptibility of the dermatophytic infected patients and to look for the sensitivity of the most commonly used antifungal drugs.

\section{Materials and Methods}

This study was a prospective hospital-based study conducted at NMCTH, Birgunj for a period of twelve months from date September 2017 to August 2018. The proposal of this study was submitted and approved by the Institutional Review Committee (IRC) with the number NMC/210/074/075. All patients attending skin OPD with suspected lesions of tinea infection. On the basis of clinical diagnosis in the outpatient department of dermatology, 349 cases were selected. Selected patients were thoroughly informed about the research and were assured of confidentiality. Consents were taken for the questionnaire and photograph and those who were willing to give consent were selected. Clinical data of patients were recorded on proforma. Required investigations were performed for the cases as per proforma. Skin scraping from the active margin, nail clipping, and hair plucking of suspected lesions were taken and $\mathrm{KOH}$ mount was done as shown in picture 1 followed by culture in Department of Microbiology. Culture was labeled negative, if no growth was observed at the end of 4 weeks. Disk-Diffusion Method was used to determine the sensitivity for the commonly used antifungal drugs (Clotrimazole, Fluconazole, and Itraconazole). Data was collected by questionnaire method, focusing on the object of the study and was put on the structured proforma. On receiving a case, fulfilling the inclusion criteria, the patient was explained about the study in detail. Patient and patient party was assured of confidentiality and an informed written consent was taken. Data was entered in Excel master sheet with coding of the variables. SPSS was used for the evaluation. Chi square test and contingency table were used as significant test analysis. Findings were presented as tables, bar diagrams, and pie charts. Frequencies and percentages were calculated.

\section{Results}

Out of 349 patients, 215 were male and 134 were female. Male to female ratio was 1.6:1. The youngest patient was 1 year old and the oldest patient was 70 years old. Majority of the patient belonged to the age group $20-29$ years, i.e. $27.3 \%$ as shown in Figure 1 . The most common variant was tinea corporis accounting for $38.1 \%$, followed by multiple types of infection 83 cases, i.e. $23.7 \%$ as shown in Figure 2. As seen from Table 1 the maximum duration of illness was 5 weeks to 20 weeks, thus it may favour that the infection runs a chronic course. Family history was positive in nearly $40 \%$ as seen in Table 2 showing the importance of person to person transmission as well as treatment of all family members simultaneously. On treatment history, the maximum patient had already used OTC products which mainly contain steroid only or a combination cream, i.e., shown in Table 3, and these OTC products not only prolong the duration of illness but also have side effects like atrophy, striae, contact dermatitis etc.

Tables 4, 5, 6 illustrates $\mathrm{KOH}$ positive was seen in 65.3\% and culture positive was seen in $57.9 \%$. $\mathrm{KOH}$ positive and culture positive was seen in $29.2 \%$ (102 cases). $\mathrm{KOH}$ positive and culture negative was seen in $36.4 \%$ (127 cases). $\mathrm{KOH}$ negative and culture negative in 5.7\% (20 cases) and $\mathrm{KOH}$ negative and culture positive in $28.7 \%$ (100 cases).

Trichophyton rubrum was the dominant species of fungus isolated followed by $T$. mentagrophytes as shown in Table 7. Itraconazole was the most sensitive drug and fluconazole as the least sensitive and most resistant drug against the isolated dermatophyte species as shown in Figure 3.

Figure 4, 5a and 5b illustrates the $\mathrm{KOH}$ mount showing branching fungal hyphae and isolation of colony and tear drop microconidia after culture respectively. Figure 6 illustrates the disk diffusion test showing the sensitivity of the itraconazole in comparison to other tested antifungals. 
Table 1: Distribution of cases according to duration of disease

\begin{tabular}{|l|c|c|}
\hline \multicolumn{1}{|c|}{ Duration in Weeks } & Frequency & Percent \\
\hline Below 5 & 114 & 32.7 \\
\hline $5-9$ & 82 & 23.5 \\
\hline $10-14$ & 42 & 12.0 \\
\hline $15-19$ & 12 & 3.4 \\
\hline Above 20 & 99 & 28.4 \\
\hline Total & $\mathbf{3 4 9}$ & $\mathbf{1 0 0}$ \\
\hline
\end{tabular}

Table 2: Family history

\begin{tabular}{|l|c|c|}
\hline Family History & Percentage & Number of cases \\
\hline Positive & $39.9 \%$ & 139 \\
\hline Negative & $60.1 \%$ & 210 \\
\hline Total & $\mathbf{1 0 0 . 0 \%}$ & $\mathbf{3 4 9}$ \\
\hline
\end{tabular}

Table 3: Treatment history

\begin{tabular}{|c|c|c|}
\hline Topical Treatment & Percentage & Number of Cases \\
\hline $\begin{array}{l}\text { Steroid alone or combination creams (with antifungal } \\
\text { antibacterial or salicylic acid) }\end{array}$ & $60.6 \%$ & 211 \\
\hline Antifungal creams & $20.1 \%$ & 70 \\
\hline Salicylic acid alone & $12 \%$ & 42 \\
\hline No treatment & $7.3 \%$ & 25 \\
\hline TOTAL & $100.0 \%$ & 349 \\
\hline
\end{tabular}

Table 4: Distribution of cases according to the positivity pattern of fungal hyphae by KOH mount (direct microscopy)

\begin{tabular}{|l|c|c|}
\hline KOH & Number & Percentage \\
\hline Positive & 228 & $65.3 \%$ \\
\hline Negative & 121 & $34.7 \%$ \\
\hline Total & $\mathbf{3 4 9}$ & $\mathbf{1 0 0 \%}$ \\
\hline
\end{tabular}

Table 5: Distribution of cases according to the result of Culture Positivity

\begin{tabular}{|l|c|c|}
\hline Culture & Number & Percentage \\
\hline Positive & 202 & $57.9 \%$ \\
\hline Negative & 147 & $42.1 \%$ \\
\hline Total & $\mathbf{3 4 9}$ & $\mathbf{1 0 0 \%}$ \\
\hline
\end{tabular}

Table 6: Distribution of cases according to the result of $\mathrm{KOH} \&$ Culture

\begin{tabular}{|l|c|c|c|c|}
\hline \multirow{2}{*}{ Clinical variants } & \multicolumn{2}{|c|}{ KOH mount } & Culture \\
\cline { 2 - 5 } & Positive & Negative & 6 & Pegative \\
\hline Tinea Unguium & 11 & 4 & 7 & 9 \\
\hline Tinea Capitis & 8 & 4 & 72 & 61 \\
\hline Tinea Corporis & 90 & 43 & 42 & 9 \\
\hline Tinea Cruris & 42 & 28 & 12 & 1 \\
\hline Tinea Faciei & 14 & 7 & 2 & 5 \\
\hline Tinea Manuum & 1 & 2 & 7 & 29 \\
\hline Tinea Pedis & 7 & 5 & 54 & $\mathbf{1 4 7}$ \\
\hline Multiple Type & 55 & 28 & $\mathbf{2 0 2}$ & 9 \\
\hline Total & $\mathbf{2 2 8}$ & $\mathbf{1 2 1}$ & & \\
\hline
\end{tabular}


Table 7: Clinical types of disease in relation to the species identified

\begin{tabular}{|l|c|c|c|c|}
\hline \multirow{2}{*}{ Diagnosis } & \multicolumn{3}{|c|}{ Species } & \multirow{2}{*}{ Total } \\
\cline { 2 - 5 } & M. gypseum & T. mentagrophyte & T. rubrum & 7 \\
\hline Tinea Capitis & 7 & 0 & 0 & 54 \\
\hline Mixed Type & 2 & 21 & 41 & 72 \\
\hline Tinea Corporis & 5 & 26 & 27 & 42 \\
\hline Tinea Cruris & 3 & 12 & 4 & 12 \\
\hline Tinea Faciei & 1 & 7 & 1 & 2 \\
\hline Tinea Manuum & 1 & 0 & 3 & 7 \\
\hline Tinea Pedis & 1 & 3 & 4 & 6 \\
\hline Tinea Unguium & 0 & 2 & $\mathbf{1 1 1}$ & $\mathbf{2 0 2}$ \\
\hline Total & $\mathbf{2 0}$ & $\mathbf{7 1}$ & $\mathbf{5 5 . 0 \%}$ & $\mathbf{1 0 0 . 0 \%}$ \\
\hline Percentage & $\mathbf{9 . 9 \%}$ & $\mathbf{3 5 . 1 \%}$ & & \\
\hline
\end{tabular}

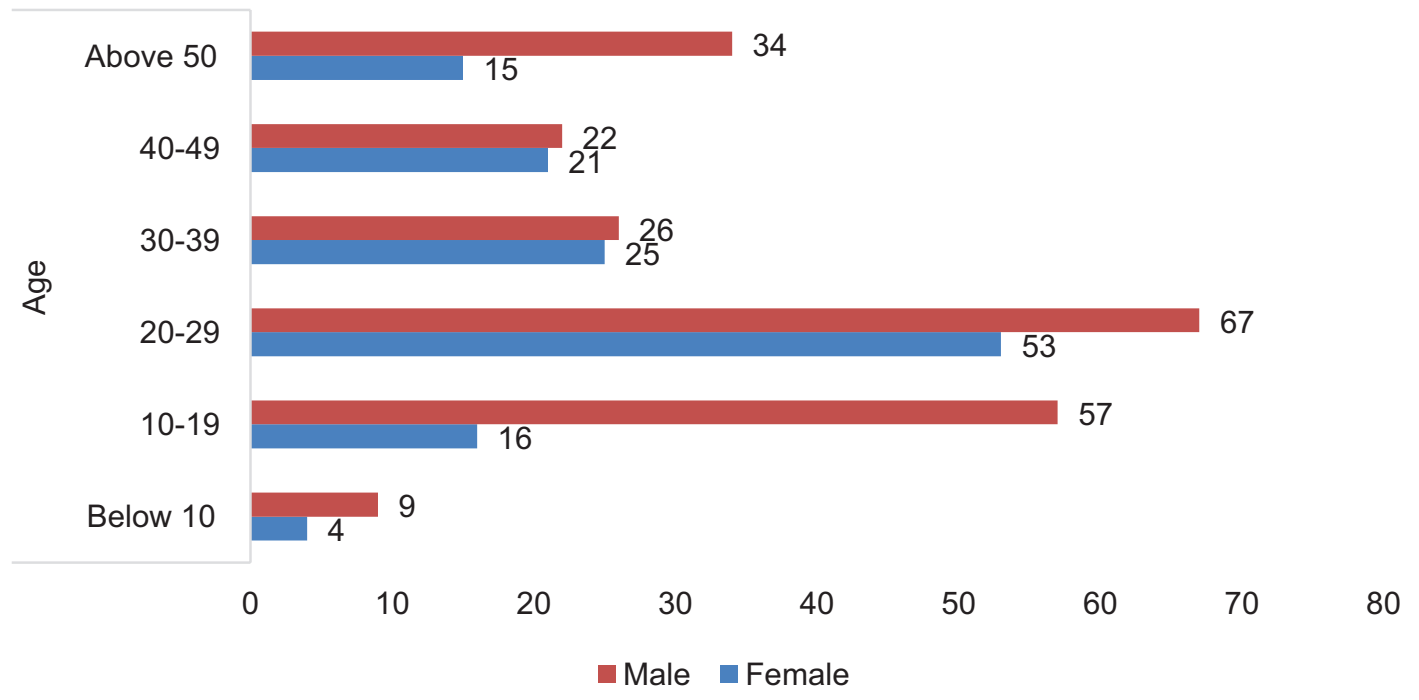

Figure 1: Gender distribution of cases according to age

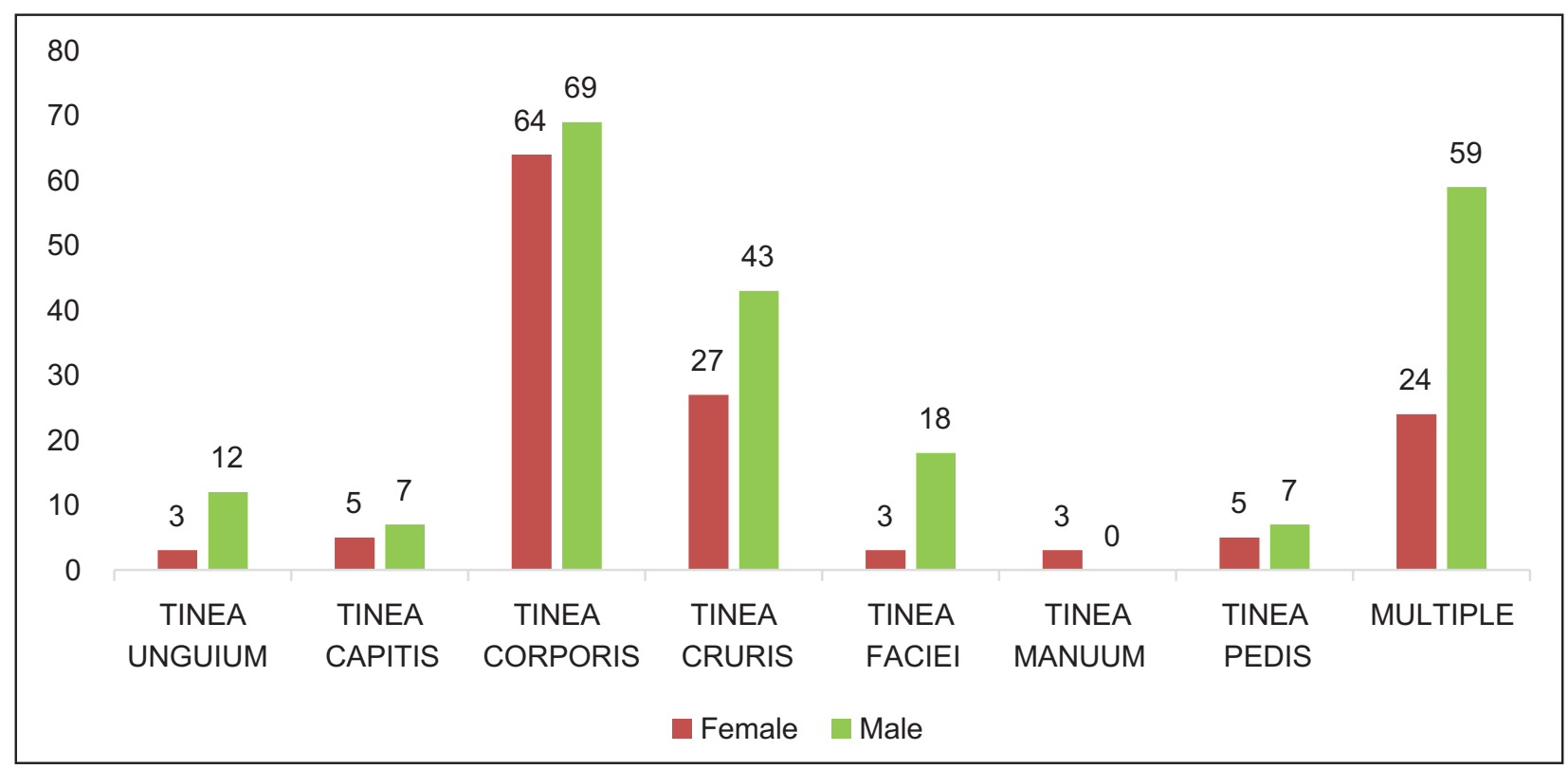

Figure 2: Diagram showing the distribution of cases according to clinical type and gender 


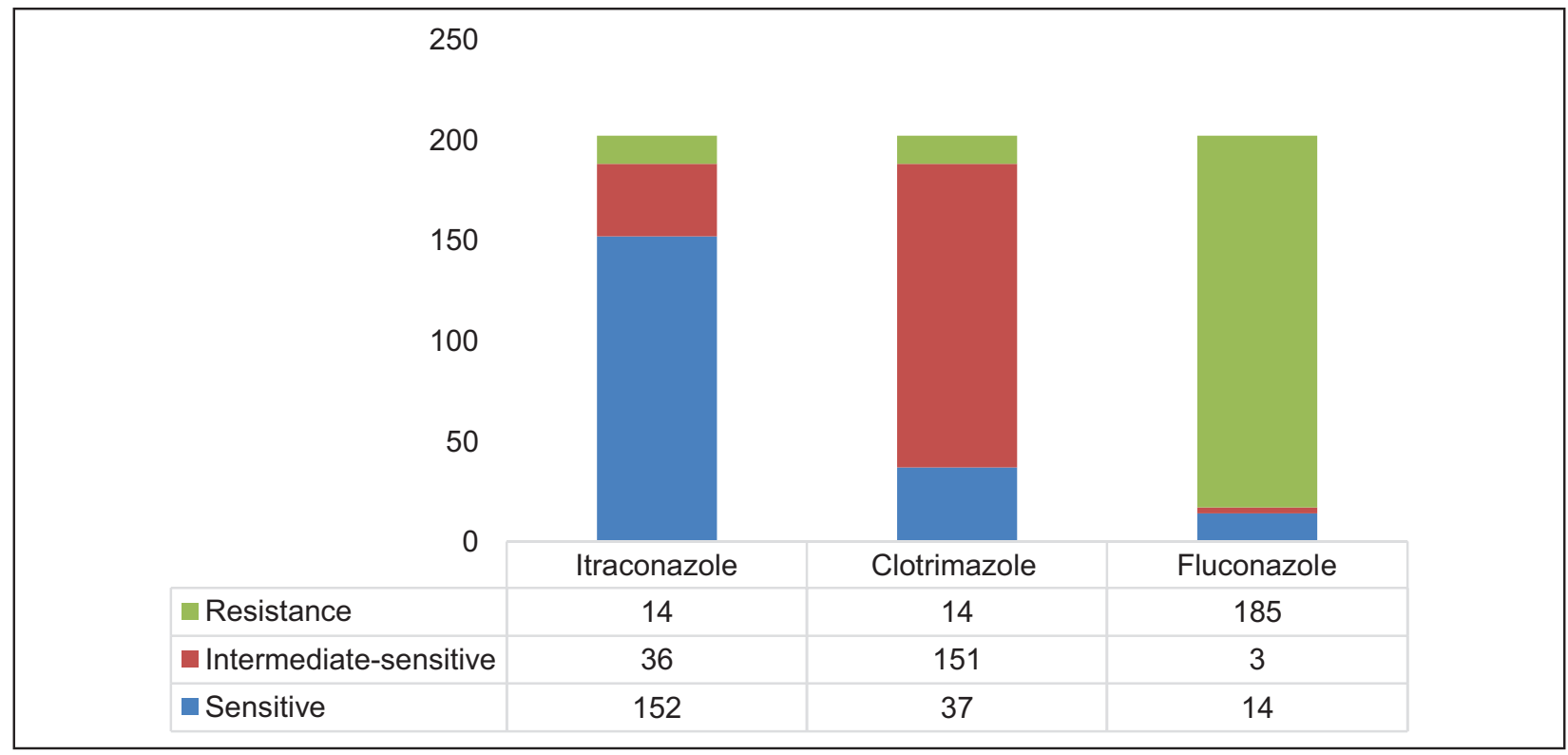

Figure 3: Pattern of sensitivity and resistance to antifungals

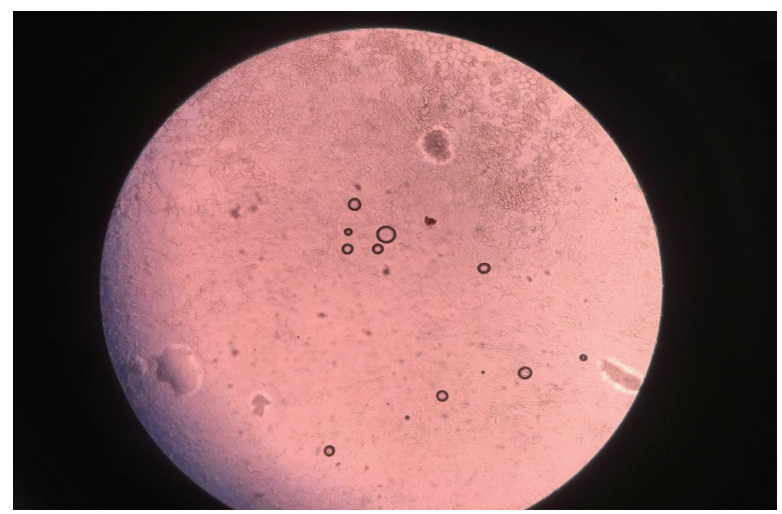

Figure 4: $\mathrm{KOH}$ mount showing branching fungal hyphae.

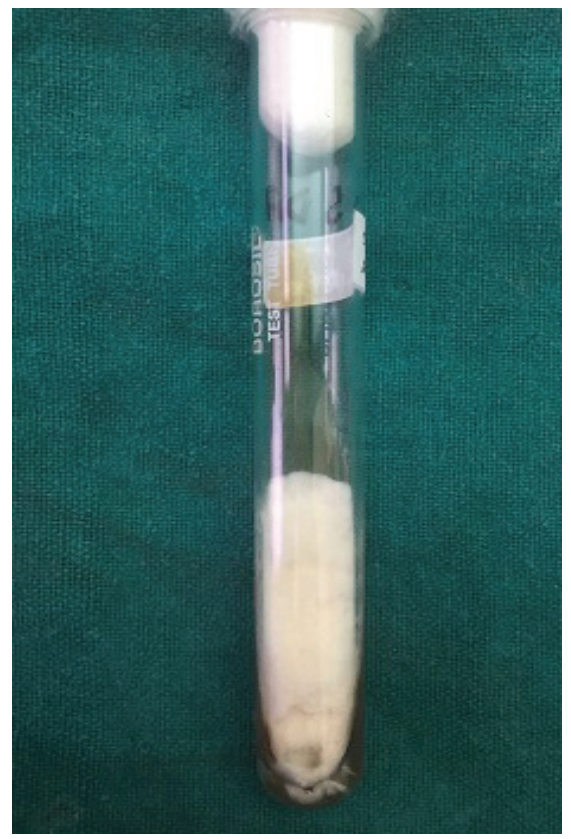

Figure 5a: Trichophyton rubrum: white colony

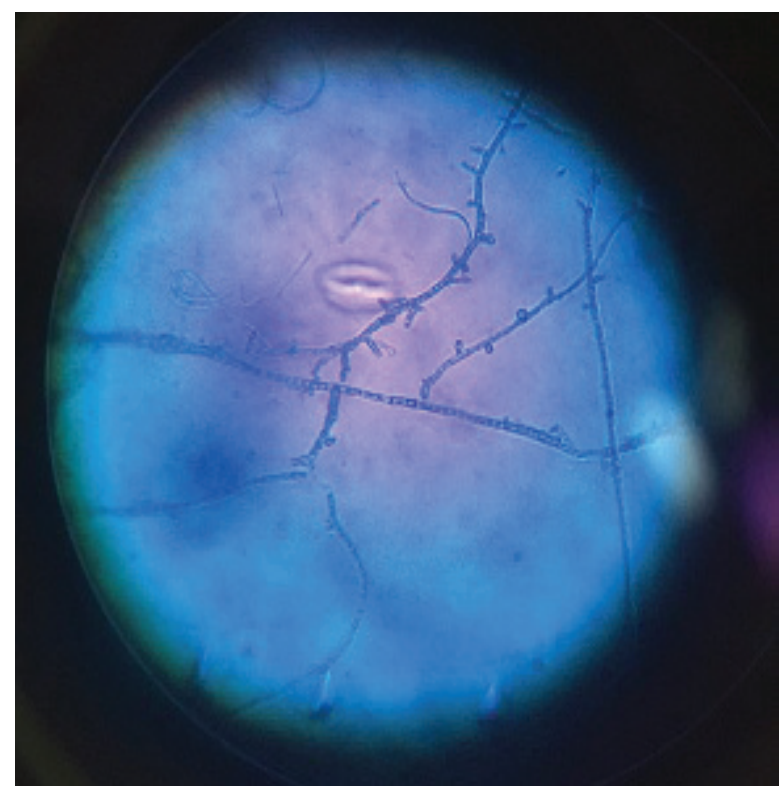

Figure 5b: tear drop microconidia

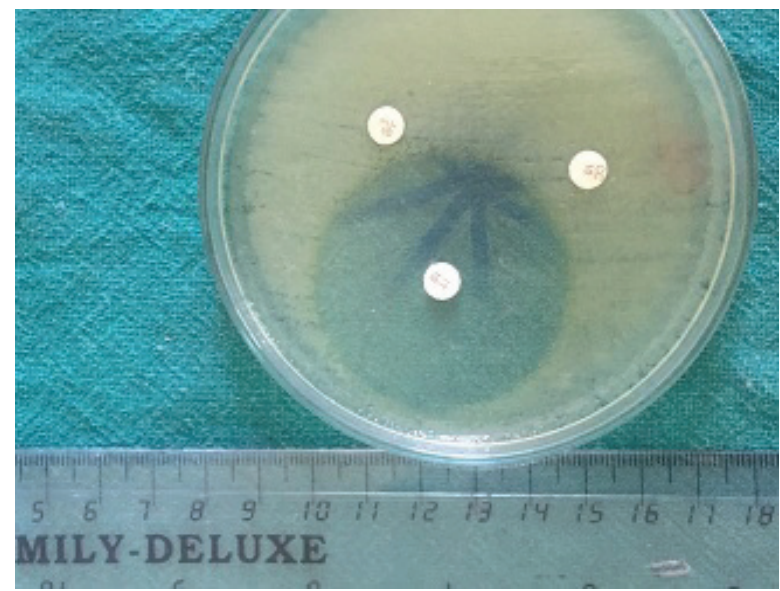

Figure 6: Disk Diffusion test showing sensitive to Itraconazole 


\section{Discussion}

Dermatophytosis has a wide geographical distribution; the species of dermatophytes causing infection may vary from region to region and are geographically restricted except some species like Trichophyton rubrum which have a cosmopolitan distribution. Present hospitalbased prospective study was carried out to evaluate the clinicomycological profile and antifungal sensitivity pattern in dermatophyte infection at Department of Dermatology, National Medical College Teaching Hospital, Birgunj, Nepal. Three hundred and forty-nine cases of dermatophytosis were analyzed to evaluate the clinical patterns, prevalence, and antifungal sensitivity of causative fungal species.

In our study, over all male (61.6\%) preponderance was observed with male to female ratio of $1.6: 1$ as demonstrated in figure 1 which is consistent to other studies, Bindu et al. ${ }^{6}$ and Bhatia et al. ${ }^{7}$ also reported male predominance. The higher prevalence of dermatophytosis in males may be due to the differences in occupational exposure as males are more involved in exhaustive physical work and prolonged exposure to sun leading to excessive sweating. In addition, tight fittings and synthetic clothing, particularly in males, provide damp, sweaty, and warm skin conditions leading to infection.

Agrawall et al. ${ }^{8}$ from Eastern Nepal reported the most common age group of tinea infection between 11 to 20 years of age and a similar result was observed by Bindu et $a .^{6}$ In contrast, the present study showed tinea infections in the age group between 20 to 29 years $(27.3 \%)$ as shown in figure 1 . The probable reason for the higher prevalence in these age groups could be that the individuals in these groups are often the most active because of their involvement in outdoor activities.

Tinea corporis (38.1\%) was the dominant clinical type of dermatophytosis observed in our study followed by mixed type $(23.7 \%)$ and tinea cruris $(20.6 \%)$ as shown in figure 2. It is consistent with other studies reported by Putta $\mathrm{S}$ et al., Balakumar S et al., Kaur R, Shrestha $\mathrm{S}$, Khadka S et al., Poluri LV et al. ${ }^{9-14}$ which also show tinea corporis as the most common infection among various clinical types.

In the present study (32.7\%) of patients reported for treatment within 5 weeks of onset of disease followed by 20 weeks (28.4\%) as shown in Table 1, which were similar to Gupta AK et al. ${ }^{15}$ This reflects the increasing public awareness amongst the citizens as well as the easy accessibility to OTC drugs and health facilities and incomplete treatment leading to relapse as shown in Table 3.

In our study, a history of fungal infections in family members was elicited in $40 \%$ of cases as elicited in Table 2 rectifying that transmission by direct contact occurs in tinea infection, explaining the transmission in family members might be due to fomites or de novo infection. Findings were consistent with that of Gupta AK et al. ${ }^{15}$, that reported $39.9 \%$ had family history positive and $60 \%$ had negative family history.

Overall, $65.3 \%$ (228 out of 349 ) cases were positive for fungal elements on direct microscopic examination $(\mathrm{KOH})$ as in tables 4, 6. Similarly, Bhatia et al. ${ }^{7}$, their study showed high positive direct microscopy. The possible reason for the high $\mathrm{KOH}$ positivity in our study could be because most of the patients attended the clinic within 5 weeks of onset of the disease before using any antifungal drugs as in Table 1.

Overall, positive culture was observed in $57.9 \%$ of cases as demonstrated in Table 5, 6. Similar findings were noted in study of Kaur R, Shrestha S, Sundar K and LV Poluri et al. as 46\%, 57.2\%, and 55.5\%. ${ }^{10-13}$ High positivity in cases of present study might be due to good laboratory support.

Present study showed T. rubrum 55\% (111 out of 202 cases) as the predominant species identified, followed by $T$. mentagrophytes (35.1\%) and M. gypseum $(9.9 \%)$ as shown in table 7. Similarly, V Bindu et al., Agarwalla A et al., Poluri LV et al. ${ }^{6,8,10}$ found T. rubrum as predominant species. The possible explanation for this may be that T. rubrum is generally linked to chronic dermatophytosis.

In vitro activity of six antifungal drugs tested against clinically important dermatophytes by Pakshir et al. ${ }^{16}$ reported susceptibility of antifungal drugs such as Ketoconazole 31 (77.5\%) sensitive, Miconazole 36 (90\%) sensitive, Clotrimazole 39 (97.5\%) sensitive and Fluconazole 39 (97.5\%) resistance. Similarly, in our study, the test results of the susceptibility to antifungal drugs were as follows: Itraconazole (75.2\%) sensitive, Clotrimazole (74.8\%) intermediate sensitive, and Fluconazole (91.6\%) resistant as elicited in figure 3.

\section{Conclusion}

Clinical findings, $\mathrm{KOH}$ mount, and culture reports were found to be complementary to each other in the diagnosis of superficial mycosis. Any clinical diagnosis needs to be supported by laboratory diagnosis. 
Culture is a necessary adjunct to direct microscopic examination for definitive identification of the etiological agents. The treatment of fungal infection would be more effective when antifungal therapy is based on the identification of fungal isolates. The increasing resistance of antifungal drugs, which may be responsible for the exceeding cause of treatment failure in dermatophyte infections. Such increasing fungal resistance is quite a challenge to the field of medicine, which demands the wise selection of antifungals by the physicians.

\section{References}

1. Hainer BL. Dermatophyte infections. Am Fam Physician 2003;67(1):101-10.

2. Ameen M. Epidemiology of superficial fungal infections. Clin Dermatol 2010;28(2):197$201 . \quad$ https://doi.org/10.1016/j. clindermatol.2009.12.005

3. Havlickova B, Czaika VA, Friedrich M. Epidemiological trends in skin mycoses worldwide. Mycoses 2008 Sep;51 Suppl 4:2-15 https://doi. org/10.1111/j.1439-0507.2008.01606.x

4. Drake LA, Dinehart SM, Farmer ER, Goltz RW, Graham GF, Hordinsky MK, et al. Guidelines of care for superficial mycotic infectionsof the skin: Tinea corporis, tinea cruris, tinea faciei, tinea manuum, and tinea pedis. J Am Acad Dermatol 1996;34(2):282-6. https://doi.org/10.1016/ S0190-9622(96)80137-X

5. Esteban A, Abarca M, Cabanes F. Comparison of disk diffusion method and broth microdilution method for antifungal susceptibility testing of dermatophytes. Medical mycology 2005;43(1):61-

6. https://doi.org/10.1080/13693780410001711 972

6. Bindu V, Pavithran K. Clinico-mycological study of dermatophytosis in Calicut Indian J Dermatol Venereol Leprol 2002;68(5):259

7. Bhatia VK, Sharma PC. Epidemiological studies on dermatophytosis in human patients in Himachal Pradesh, India. Springer plus 2014;3(1):134. https://doi.org/10.1186/2193-1801-3-134

8. Agarwalla A, Jacob M, Sethi M, Parija SC, Singh NP. A Clinico-Mycological Study of Dermatophytoses in Nepal. J Dermatol 2001;28(1):16-21. https:// doi.org/10.1111/j.1346-8138.2001.tb00080.x

9. Balakumar S, Rajan S, Thirunalasundari T, Jeeva S. Epidemiology of dermatophytosis in and around

Tiruchirapalli, Tamilnadu, India. Asian Pac J Trop Dis 2012;2(4):286-9. https://doi.org/10.1016/ S2222-1808(12)60062-0

10. Poluri LV, Indugula JP, Kondapaneni SL. Clinicomycological study of dermatophytosis in South India. J Lab Physicians 2015;7(2):84. https://doi.org/10.4103/0974-2727.163135

11. Khadka S, Sherchand JB, Pokharel DB, Pokhrel BM, Mishra SK, Dhital S, et al. Clinicomycological Characterization of Superficial Dermatophytosis from a Tertiary Care Hospital in Nepal. Dermatol Res and Prac 2016.9509705 https://doi. org/10.1155/2016/9509705

12. Mathur M, Shrestha S. Clinicomycological Profile and Antifungal Sensitivity Pattern of Commonly Used Azoles in Dermatophytosis. J Nepal Med Assoc 2015;53(198). https://doi.org/10.31729/ jnma. 2771

13. Kaur R, Panda PS, Sardana K, Khan S. Mycological pattern of dermatodermatophytosis in a tertiary care Hospital. J Trop Med 2015;2015. https://doi. org/10.1155/2015/157828

14. Putta SD, Kulkarni VA, Bhadade AA, Kulkarni VN, Walawalkar AS. Prevalence of dermatophytosis and its spectrum in a tertiary care hospital, Kolhapur. Indian Journal of Basic and Applied Medical Research 2016;5(3):595-600.

15. Gupta AK, Mohan A, Singh SK, Pandey AK. Studying the clinic mycological pattern of the dermatophytic infection attending OPD in tertiary care hospital in eastern Uttar Pradesh and Bihar. Int J Res Dermatol 2018;4(2):11825. https://doi.org/10.18203/issn.2455-4529. IntJResDermatol20180987

16. Pakshir K, Bahaedinie L, Rezaei Z, Sodaifi M, Zomorodian $\mathrm{K}$. In vitro activity of six antifungal drugs against clinically important dermatophytes. Jundishapur Journal of Microbiology. 2009;2(4):158-63. 